\title{
Gutted adenoviral vector growth using E1/E2b/E3-deleted helper viruses
}

\author{
Catherine Barjot ${ }^{1 \dagger}$ \\ Dennis Hartigan- \\ O'Connor ${ }^{1,2,3}$
}

Giovanni Salvatori ${ }^{1 *}$

Jeannine M. Scott ${ }^{1,3}$

Jeffrey S. Chamberlain ${ }^{1,2,3 *}$

${ }^{1}$ Department of Human Genetics, University of Michigan, Ann Arbor, MI, USA

${ }^{2}$ Program in Cellular and Molecular Biology, University of Michigan, Ann Arbor, MI, USA

${ }^{3}$ Department of Neurology, University of Washington School of Medicine, HSB Room K243, Box 357720 ,

Seattle, WA 98195-7720, USA

*Correspondence to:

Dr Jeffrey S. Chamberlain, University of Washington School of Medicine, Department of Neurology, Box 357720, 1959 N.E. Pacific Street, Seattle, WA 98195-7720, USA.

E-mail: jsc5@u.washington.edu

†Current address: UMR INRA 703, ENVN - Atlanpole La Chantrerie, BP 40706, 44307 Nantes Cedex 3, France.

†Current address: Department of Immunology, Sigma-Tau, 00040 Pomezia, Italy.
Received: 12 March 2002

Revised: 22 April 2002

Accepted: 3 May 2002

\begin{abstract}
Background Helper-dependent, or gutted, adenoviruses (Ad) lack viral coding sequences, resulting in reduced immunotoxicity compared with conventional Ad vectors. Gutted Ad growth requires a conventional Ad to supply replication and packaging functions in trans. Methods that allow high-titer growth of gutted vectors while reducing helper contamination, and which use safer helper viruses, will facilitate the use of gutted Ad vectors in vivo.
\end{abstract}

Methods Replication-defective helper viruses were generated that are deleted for Ad E1, E2b and E3 genes, but which contain loxP sites flanking the packaging signal. Complementing Ad packaging cell lines (C7-cre cells) were also generated by transfecting 293 cells with the Ad E2b genes encoding DNA polymerase and pre-terminal protein, and with a cre-recombinase plasmid.

Results We show that C7-cre cells allow efficient production of gutted Ad using $\Delta \mathrm{E} 1+\Delta \mathrm{E} 2 \mathrm{~b}+\Delta \mathrm{E} 3$ helper viruses whose growth can be limited by creloxP-mediated excision of the packaging signal. Gutted Ad vectors carrying $\sim 28 \mathrm{~kb}$ cassettes expressing full-length dystrophin were prepared at high titers, similar to those obtained with E2b+ helpers, with a resulting helper contamination of $<1 \%$.

Conclusions These new packaging cell lines and helper viruses offer several significant advantages for gutted Ad vector production. They allow gutted virus amplification using a reduced number of passages, which should reduce the chances of selecting rearranged products. Furthermore, the residual helper contamination in gutted vector preparations should be less able to elicit immunological reactions upon delivery to tissues, since E2b-deleted vectors display a profound reduction in viral gene expression. Copyright $(\odot$ 2002 John Wiley \& Sons, Ltd.

Keywords adenovirus; gutted adenovirus; helper virus; Duchenne muscular dystrophy; dystrophin; gene therapy

\section{Introduction}

Gene therapy applications of adenoviral vectors have been extensively explored. Despite great progress, shortcomings in vector production, immunogenicity and cloning capacity have imposed significant limitations on their use in vivo [1]. The development of helper-dependent (hdAd) or "gutted" adenoviral vectors, whereby the sequences and regulatory elements of a therapeutic transgene are flanked by the minimal adenovirus sequences required for replication and packaging, holds great promise to overcome 
many of these limitations [2-4]. One potential problem with the gutted Ad system is that vector amplification requires simultaneous replication of a helper virus to provide proteins necessary for replication and encapsidation in trans, leading to co-production of helper and gutted viruses [5]. To produce gutted Ad stocks free of helper virus, several laboratories have used the cre-recombinase-loxP system [6,7]. In general, the helper virus packaging sequence is flanked by loxp sites and is efficiently excised by cre recombinase during growth in vitro, yielding an unpackagable helper genome that still produces Ad proteins needed for helper activity. Despite the efficiency of the cre-loxp system to reduce helper contamination, gutted virus preparations are never completely helper free $[6,8,9]$, an obstacle to their therapeutic utility. Helper virus genomes that are delivered to the target tissue may lead to undesirable expression of viral genes, which at certain threshold levels can elicit a host immune response [10-12]. An early gene product required for expression of the late viral proteins is the E2b-encoded Ad DNA polymerase (Pol; [13]). We reasoned that removal of the Pol gene would result in a safer helper virus less able to generate replication-competent Ad (RCA) during growth.

Growth of polymerase-deleted ( $\Delta$ Pol) Ad vectors can be performed efficiently on C7 cells, which express both Ad Pol and pre-terminal protein (pTP) $[13,14]$. Conveniently, these C7 cells have also been shown to facilitate greatly the preparation of gutted Ad genomes from plasmid substrates $[15,16]$. To enable efficient production of gutted Ad vectors with minimal levels of contamination by an E2b-deleted helper virus, we constructed a helper virus with loxp sites flanking the packaging signal on an Ad genome deleted for the Pol gene. Together with E1 and E3 deletions, polymerase activity depletion has been shown to almost completely eliminate expression of viral late genes in non-complementing cells by blocking viral DNA replication [13]. Furthermore, in immunocompetent mice, the persistence of viral DNA from an E1- and polymerase-deleted virus carrying a lacZ gene was extended, leading to an increase in the duration of transgene expression compared to a virus carrying the same transgene but having only the E1 deletion [17]. To grow gutted vectors with these new helper viruses, we generated modified C7 cell lines that express cre recombinase.

We demonstrate that the use of cre-expressing C7 cells enhances the amount of gutted virus rescued using a transfection/infection procedure. This improvement leads to high titers of gutted virus as early as the second or third passage after rescue. Our results suggest that the use of the cre-loxp system and a helper virus with deletions of both E1 and polymerase activity, combined with a reduced number of passages, should improve the safety of the gutted system and simplify growth of gutted Ad vectors.

\section{Materials and methods}

\section{C7-cre cell lines}

C7 cells [18] were co-transfected with $10 \mu \mathrm{g}$ of pOG231 (expressing cre recombinase; kindly provided by Dr. S. O'Gorman; [19]), and $1 \mu \mathrm{g}$ of pcDNA3 (for neomycin resistance; Invitrogen). Cre-recombinase expression from pOG231 is driven by the CMV promoter and a synthetic intron. Prior to transfection, the plasmids were linearized with PvuII and NotI, respectively. C7 cells were transfected using the calcium phosphateDNA precipitation method and stable transfectants were selected for and subcloned by colony isolation in medium supplemented with $1 \mathrm{mg} / \mathrm{ml} \mathrm{G} 418$. All reagents for cell culture were purchased from GibcoBRL. Isolated cell lines were cultured in Dulbecco's modified Eagle's medium (DMEM) supplemented with $10 \%$ fetal bovine serum in the presence of $5 \mathrm{U} / \mathrm{ml}$ each penicillin and streptomycin, plus $0.5 \mathrm{mg} / \mathrm{ml} \mathrm{G} 418$ to maintain cre expression and $100 \mu \mathrm{g} / \mathrm{ml}$ hygromycin B for Ad Pol and pTP expression.

Cre-recombinase activity in the subcloned lines was initially compared following transient transfection with pGKint $\beta$ gal. This plasmid contains a polyadenylation site flanked by loxP sites located between the PGK promoter and the $\beta$-galactosidase ( $\beta$-gal) cDNA. In the presence of cre recombinase, the internal polyadenylation sequence is excised, allowing expression of $\beta$-gal. The relative levels of cre-recombinase activity in each cell line were estimated by monitoring $\beta$-gal expression after transfection with pGKint $\beta$ gal [20]. Clones displaying the highest $\beta$-gal expression were further subcloned and expanded for additional analyses.

\section{Construction of helper viruses}

The human placental alkaline phosphatase (hpAP) cDNA and SV40 large T intron and poly(A) site were subcloned from pRSVhAPT40 (a gift from Dr. Gary Nabel) downstream of the ecdysone-inducible promoter in pIND (Invitrogen). The promoter-cDNA cassette was then excised and inserted into pABS.4 (Microbix) along with a $1.8 \mathrm{~kb}$ EcoRI stuffer fragment isolated from human dystrophin intron 45 (J. S. Chamberlain, unpublished). The resulting PacI fragment (which included a kanamycinresistance gene derived from pABS.4) was inserted into the E3 deletion site of pBHG10 (Microbix), then the kanamycin resistance gene was removed by ClaI digestion to generate pL60. To generate a virus from pL60, C7 cells were co-transfected with $5 \mu \mathrm{g}$ of supercoiled pL60 and $5 \mu \mathrm{g}$ of EcoRI-digested pAdAscL, a leftend Ad5 shuttle vector containing genome sequences from map units (mu) $0-1$ and 9-40 (M. Hauser and J. S. Chamberlain, unpublished). The resulting E1/E3deleted Ad virus (AdL60) contained the ecdysoneinducible hpAP reporter gene cassette at the site of the E3 deletion (Figure 1A). 


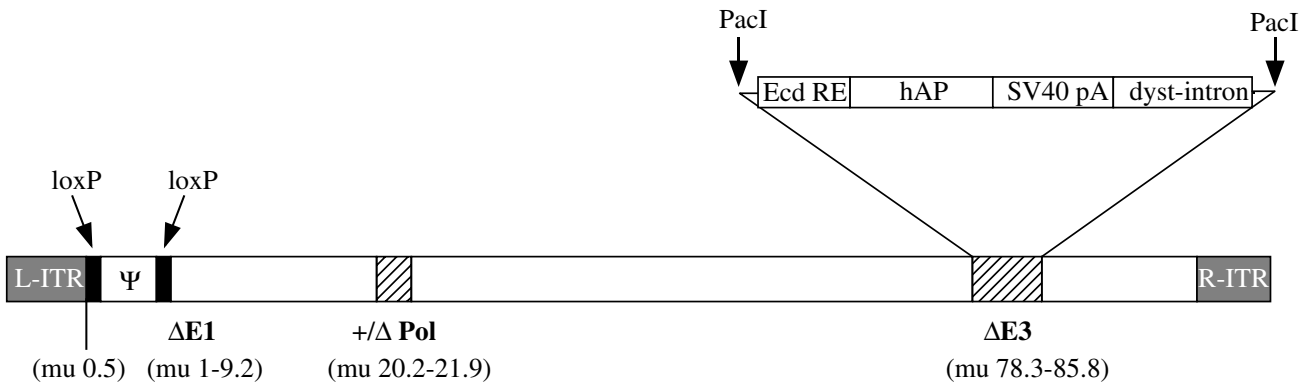

B

\begin{tabular}{|c|c|c|c|}
\hline L-ITR & $\Psi$ Ecd RE - lacZ & MCK - Mouse Dystrophin cDNA & R-ITR \\
\hline
\end{tabular}

Figure 1. Structure of helper and gutted viruses. (A) Structure of helper adenoviruses indicating features that are relevant to this study: the viral DNA packaging signal $(\Psi)$ is flanked by loxP sites $[(+)$ loxP], the human placental alkaline phosphatase (AP) reporter gene is driven by an ecdysone-inducible promoter, and the E1 and E3 regions are deleted. Dyst-intron refers to a 1.8-kb fragment from human dystrophin intron 45 that was used as a stuffer. Also shown is the location of the viral DNA polymerase gene region deleted in $\Delta$ Pol viruses (mu 20.2-21.9), and the left and right inverted terminal repeats (L- or R-ITR). The vector shown represents $\mathrm{Ad}(+) \operatorname{lox} \mathrm{P}(+)$ PolAP (see text); the same vector lacking the Pol gene is referred to as $\mathrm{Ad}(+) \operatorname{loxP} \Delta \mathrm{PolAP}$. The total size of the helper viruses is $\sim 36 \mathrm{~kb}$. (B) GE $\beta$ Dys is a 28 -kb gutted adenovirus containing a $21-\mathrm{kb}$ expression cassette composed of the full-length 13.9-kb murine dystrophin cDNA regulated by the 6.5-kb mouse muscle creatine kinase (MCK) promoter/enhancer, an ecdysone-inducible $\beta$-gal reporter gene, and one loxP site between the left ITR and the viral DNA packaging signal (G. Salvatori, M. Hauser, J. S. Chamberlain et al., manuscript in preparation)

LoxP sites were added on either side of the packaging signal by ligating synthetic double-stranded oligonucleotides containing loxP sites into the AflIII (0.5 mu) and BglII (1 mu) sites of plasmid pAdBglII to form pAdloxPBglII. $10 \mu \mathrm{g}$ of this 'floxed' left-end shuttle plasmid were linearized by digestion with NheI and co-transfected into C7 cells with $1 \mu \mathrm{g}$ of ClaI-digested viral DNA isolated from adenoviral strains dl7001 or dl7001 $\Delta$ Pol [13] to create viruses AdloxP $(+)$ Pol7 and AdloxP $\Delta$ Pol7. DNA $(1 \mu \mathrm{g})$ from each of these two viruses was then digested with EcoRI and AseI and separately co-transfected with $1 \mu \mathrm{g}$ of BclI-digested AdL60 genomic DNA into C7 cells to generate the two helper viruses $\mathrm{Ad}(+) \operatorname{lox} \mathrm{P}(+) \mathrm{PolAP}$ and $\mathrm{Ad}(+)$ loxP $\triangle \mathrm{PolAP}$ (Figure 1A). Sixteen hours posttransfection, the cells were passaged and replated with fresh, untransfected C7 cells and distributed into 24-well plates as described previously [13]. Cells that displayed evidence of virus-induced cytopathic effect (CPE) were transferred into 35-mm dishes for further replication. When these cells showed complete CPE, the cells and medium were harvested and divided: $75 \%$ was used for extraction of viral DNA, and $25 \%$ was saved for future expansion. Clones carrying the desired recombinant virus, as determined by Southern analysis, were plaque-purified prior to large-scale expansion as described previously [18].

\section{Colorimetric assays to determine viral transducing unit titer}

Infected cell lysates were diluted in $500 \mu \mathrm{l}$ complete medium containing $5 \mu \mathrm{M}$ ponasterone A (Invitrogen), an analogue of ecdysone, and used to infect monolayers of EcR-293 cells (Invitrogen) in a poly-L-lysine-coated 24-well tissue culture plate (Biocoat). After $16 \mathrm{~h}$, the medium was removed, the cells were washed twice with phosphate-buffered saline (PBS) and fixed with $0.5 \%$ glutaraldehyde in PBS, and stained overnight for either alkaline phosphatase or $\beta$-gal expression, as described [3]. The number of cells that stained positively for $\beta$-gal or alkaline phosphatase activity was used to calculate viral titers as transducing units per $\mathrm{ml}(\mathrm{tu} / \mathrm{ml})$.

\section{Quantitative PCR}

Purified virus stocks were quantified by real-time PCR, using primers and a probe for the mouse muscle creatine kinase (MCK) gene promoter for the gutted virus, and for the Ad L2 gene for the helper. Primer sequences were: MCK forward, 5'-CGCAACGAAGCTATGTCCAA3'; MCK reverse, 5'-GCTTGTAATCCTGCTCTTCCTTCTT3'; MCK probe, 5'-VIC-CAGGTCATCGCGCCGGAGATCTATAMRA-3'; L2 forward, 5'-CGCAACGAAGCTATGTCCAA3'; L2 reverse, 5'-GCTTGTAATCCTGCTCTTCCTTCTT3'; L2 probe, 5'-VIC-CAGGTCATCGCGCCGGAGATCTATAMRA-3'. Prior to PCR amplification, virion capsids were destroyed by digestion with proteinase $\mathrm{K}$ at $37^{\circ} \mathrm{C}$ for $1 \mathrm{~h}$, followed by inactivation at $95^{\circ} \mathrm{C}$ for $20 \mathrm{~min}$. Serial dilutions of the digested samples were used as template in the PCRs and were compared with standards of known quantity. All samples were amplified and fluorescence of the reporter dyes recorded using the Applied Biosystems 7700 sequence detection system. 


\section{Southern analysis of cre-recombinase activity on viral DNA substrates}

C7 and C7-cre cells were infected in parallel with equal volumes of cell lysate or banded virus from Ad(+) loxP(+)PolAP infected C7 cells. After $48 \mathrm{~h}$, total viral DNA was extracted by the method of Hirt [21], digested with HindIII, and separated on a $0.8 \%$ agarose gel. The resolved fragments were transferred to a nylon membrane and hybridized with a probe from the left end of the virus that had been radiolabeled with $\alpha^{32} \mathrm{P}$-dCTP by random-priming. Digestion of wildtype viral DNA generates a fragment of $3371 \mathrm{bp}$ which contains the viral DNA packaging signal. Crerecombinase-mediated excision of the sequences between the loxp sites, which includes the packaging signal, results in a band of $3100 \mathrm{bp}$. Hybridized bands were visualized using a phosphorimager and quantified with Imagequant software (Molecular Dynamics).

\section{Growth of gutted virus}

To initiate production of the gutted adenovirus GE $\beta$ Dys (Figure 1B; G. Salvatori, M. Hauser and J. S. Chamberlain, in preparation), C7-cre cells were plated at $2.5 \times 10^{6}$ cells $/ \mathrm{ml}$ in a $60-\mathrm{mm}$ dish and cultured to $70 \%$ confluency. The cells were then transfected with $10 \mu \mathrm{g}$ of pGE $\beta$ Dys (the GE $\beta$ Dys viral genome contained in a plasmid backbone) in the presence of $0.1 \mathrm{mM}$ chloroquine. After $4 \mathrm{~h}$ at $37^{\circ} \mathrm{C}$, the cells were subjected to a 30-s shock with 15\% glycerol in 1X HEPES-buffered saline, pH 7.05. The cells were immediately washed twice with warm PBS and then incubated at $37^{\circ} \mathrm{C}$ in tissue culture medium. At $18 \mathrm{~h}$ post-transfection, the cells were infected with either $\mathrm{Ad}(+) \operatorname{lox} \mathrm{P}(+) \mathrm{PolAP}$ helper virus at a multiplicity of infection (moi) of 1 or $\mathrm{Ad}(+) \operatorname{lox} \mathrm{P} \triangle \mathrm{PolAP}$ helper virus at a moi of 5 . The cells were harvested when they displayed complete CPE (usually 10-15 days later for C7-cre8.2 cells, and 5-7 days for C7-cre8 cells) and gutted and helper virus titers were determined using the $\beta$-gal or alkaline phosphatase assays described above. Cell lysates containing infectious gutted virus were then used in subsequent passages for virus amplification and to determine optimal conditions for gutted Ad vector growth. The amount of lysate necessary to infect more fresh cells was calculated according to the titers of the gutted and helper viruses. For the first passage, one-third of the lysate was typically used to infect a fresh 60-mm dish of C7-cre cells to keep the moi of the input gutted virus as high as possible. For subsequent passages, an input gutted virus moi of 1-2 tu/cell was used. For each passage, purified helper virus was added to the lysate to achieve a final input helper virus moi of 5 , except for the final passage, for which helper virus was added at a moi of 3 . After each round of amplification, the cells and medium were harvested when the cells displayed complete CPE, usually 5-7 days after inoculation (for C7-cre8.2 cells; 3-5 days for C7-cre8 cells).

\section{Purification of gutted virus}

After three or four serial passages, the yield from a typical gutted virus preparation approached $10^{11}$ tu in a crude cell lysate. When complete CPE was observed in the final passage, the cells were collected from the plates, pelleted at $150 \mathrm{~g}$ for $10 \mathrm{~min}$ at $4{ }^{\circ} \mathrm{C}$ and resuspended in two cell pellet volumes of $10 \mathrm{mM}$ Tris- $\mathrm{HCl}, \mathrm{pH}$ 8.0, $1 \mathrm{mM}$ $\mathrm{MgCl}_{2}$. Cell membranes were disrupted by three rounds of freeze/thaw followed by three rounds of sonication, for 1 min each, using a model 550 sonic dismembrator (Fisher Scientific) at level 5 with a microprobe. The lysate was then clarified by centrifugation at $1500 \mathrm{~g}$ for $10 \mathrm{~min}$ at $4{ }^{\circ} \mathrm{C}$. The supernatant was stored on ice while the resulting pellet of cell debris was resuspended in $10 \mathrm{ml}$ of $10 \mathrm{mM}$ Tris- $\mathrm{HCl}, \mathrm{pH} 8.0,1 \mathrm{mM} \mathrm{MgCl}_{2}$, and subjected to three additional rounds of sonication. The supernatants from the clarified lysates were then pooled and treated with $50 \mu \mathrm{g} / \mathrm{ml}$ each of DNaseI and RNaseA to degrade unpackaged viral and cellular nucleic acids. Both gutted and helper viruses were co-purified on a $\mathrm{CsCl}$ step gradient of $25 \% 1.4 \mathrm{~g} / \mathrm{ml} \mathrm{CsCl}$ and $50 \% 1.3 \mathrm{~g} / \mathrm{ml} \mathrm{CsCl}$. The remaining $25 \%$ included the prepared virus solution brought to $1.1 \mathrm{~g} / \mathrm{ml}$ with $\mathrm{CsCl}$ before layering onto the gradient. The gradients were centrifuged at $53000 \mathrm{~g}$ for $4-16 \mathrm{~h}$ at $4{ }^{\circ} \mathrm{C}$ in a Beckman SW-28 rotor. The gutted and helper viruses were not separated on this gradient but were subsequently resolved on a self-forming gradient of $1.34 \mathrm{~g} / \mathrm{ml} \mathrm{CsCl}$ centrifuged for $12 \mathrm{~h}$ at $320000 \mathrm{~g}$ for $12 \mathrm{~h}$ followed by $73000 \mathrm{~g}$ at $4^{\circ} \mathrm{C}$ in a Beckman NVT65 rotor. The viral band was then pulled from the gradient using an 18-gauge needle, and in the case of gutted virus, further purified from helper virus through one or two additional self-forming gradients. Purified virus was dialysed against 10 mM HEPES buffer, pH 7.6, containing 5\% sucrose. Viral titers were determined using the colorimetric and real-time PCR assays as described above.

\section{Results}

\section{Generation of loxP-containing, polymerase-deleted helper viruses}

The C7 cell line is a derivative of the adenovirus packaging cell line 293 that expresses the E2b-encoded polymerase and pre-terminal proteins of Ad5 [14,18,22]. As a result of the additional adenoviral proteins, C7 cells produce adenovirus by efficiently rescuing supercoiled or linear viral genome templates [15]. C7 cells also allow growth of polymerase-deleted adenoviral vectors [13]. For enhanced production of helper-dependent or "gutted" adenoviral vectors, we further modified the C7 cells by making them capable of site-specific excision of the DNA packaging signal in first-generation or helper viruses, thereby increasing the yield and simplifying purification of gutted viral vectors. For this site-specific excision, we chose the cre-loxP system [6]. 
Figure 1A illustrates the basic features of the various helper viruses we generated. AdL60 is an E1/E3-deleted virus carrying an ecdysone-inducible alkaline phosphatase expression cassette. We also generated four viruses that contain loxp sites flanking the packaging signal in an E1/E3-deleted backbone $[(+)$ loxP $]$, and which either contain or lack the Ad DNA polymerase gene [(+)Pol or $\Delta \mathrm{Pol}]$ and the inducible alkaline phosphatase reporter cassette (AP). Most of the studies described below utilized either AdL60, Ad(+)loxP(+)PolAP or Ad(+)loxP $\Delta$ PolAP (Figure 1A; see 'Materials and methods').

We next sought to determine whether our insertion of loxP sites adjacent to the viral DNA packaging signal would have any detrimental effects on the replication of the virus. We compared the viral output from C7 cells infected with a virus that either contained, or lacked, loxP sites. Figure 2 shows that the production of the $(+)$ lox $\mathrm{P}$ virus is nearly identical to the $(-)$ lox $\mathrm{P}$ virus. We concluded that the insertion sites for the loxP sequences did not impair viral replication in C7 cells.

\section{Isolation of C7-cre cells}

C7 cells were stably transfected with a cre-recombinase expression construct and subcloned as described in 'Materials and methods'. The initial transfectants were screened for their ability to excise a loxP-flanked fragment of DNA present in pGKint $\beta$ gal such that it blocks expression of the lacZ reporter gene. Proper excision of the internal fragment enabled expression of the $\beta$-gal cDNA, leading to the production of quantifiable $\beta$-gal. C7-cre subclones that produced appreciable $\beta$-gal activity

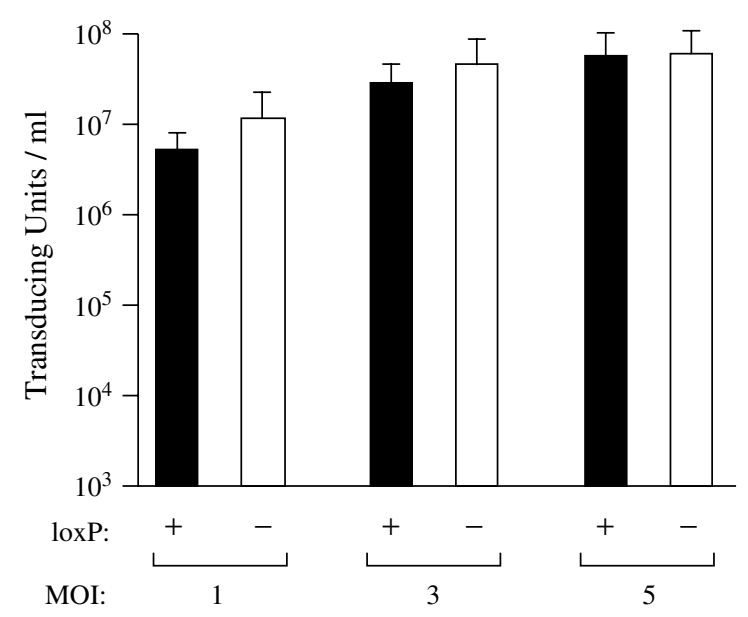

Figure 2. Virus production is not impaired by the presence of loxP sites. C7 cells were infected at $90 \%$ confluency with $\mathrm{CsCl}$ gradient-purified viruses that either contained $(+)$ or lacked $(-)$ loxP sequences on each side of the packaging signal. Viruses were added at an moi of 1,3 , or $5 \mathrm{tu}$ /cell. Cell lysates were prepared $48 \mathrm{~h}$ post-infection, and the viral titers were measured on EcR-293 cells. Shaded bars represent titers of $(+)$ loxP virus $[$ Ad( $(+) \operatorname{lox} \mathrm{P}(+) \mathrm{PolAP}]$; unshaded bars represent titers of $(-)$ loxP virus (AdL60). Error bars = SEM of three experiments. The yields of the $(+)$ and $(-)$ loxP viruses were not significantly different, as determined by Student's t-test with a $95 \%$ confidence level by this assay were expanded for additional analysis (data not shown).

The ability of these subclones to excise the loxpflanked viral packaging signal was evaluated in two ways: Southern analysis of viral DNA from infected C7-cre cells, and comparison of relative viral titers produced in C7-cre vs. C7 cells. For DNA analysis, the cells were infected with $\mathrm{Ad}(+) \operatorname{lox} \mathrm{P}(+)$ PolAP for $48 \mathrm{~h}$ followed by total DNA extraction, digestion and Southern analysis. We observed that the virus produced by the C7 cells yielded DNA with a left-end fragment of the predicted full-length size (3371 bp) that includes the packaging signal (Figure 3A). In contrast, the C7-cre clones produced viral DNA with a shorter left-end fragment (3100 bp), indicating that the packaging signal had been excised. This experiment also revealed that the individual $\mathrm{C7}$-cre subclones varied in their ability to excise packaging signals. For example, quantitative analysis of the relative intensity of the bands indicated that clones \#8 and \#25 were more efficient ( $\geq 95 \%$ ) than clone $\# 15$, which excised the packaging signal from only $\sim 35 \%$ of the viral genomes. Clone \#8 was further subcloned, and after initial testing with the pGKint $\beta$ gal expression system, four new sublines were compared with C7 and C7-cre8 cells by infection with purified virus and Southern analysis. All four of the selected sublines excised the packaging signal with $>99 \%$ efficiency (Figure 3B).

The presence of active cre recombinase in the C7cre cell lines should lead to a reduction in the yields of packaged $(+)$ lox $\mathrm{P}$ viruses. To examine viral yield we performed parallel infections of $\mathrm{C7}$ and the C7-cre cell lines with CsCl-purified $\mathrm{Ad}(+) \operatorname{lox} \mathrm{P}(+) \mathrm{PolAP}$ virus. For these studies all cell lines used had undergone a similar number of passages since their transfection with the Ad Pol and pTP constructs. The cells were infected at a moi of 3 after reaching 90\% confluency in a 60-mm dish. After $48 \mathrm{~h}$, cell lysates were prepared and viral yields determined using a colorimetric assay for alkaline phosphatase. The results showed a dramatic reduction in viral yields in the cre-recombinase-expressing cells. Cell line \#25 produced the most virus (4.1\%), while line \#8.2 produced the least (0.4\%), compared with the yield obtained in C7 cells (Figure 3C). These results correlated well with the levels of excision seen in the Southern analysis studies, indicating that the creexpressing cells efficiently reduce the amount of virus produced when that virus contains a packaging signal flanked by loxp sites.

To verify that this reduction in viral yield was due to the cre-mediated excision of the packaging signal and not to a general reduction in their ability to produce virus, we compared C7 and C7-cre cells for the ability to produce virus independent of the cre-loxP system. The cells were infected at a moi of 3 with AdL60, which is identical to the $\operatorname{Ad}(+) \operatorname{lox} \mathrm{P}(+) \mathrm{PolAP}$ virus except that it lacks loxP sites (Figure 1A); $48 \mathrm{~h}$ post-infection, the amount of virus produced by C7-cre cells was not different from the amount produced by C7 cells (Figure 3D). 
A

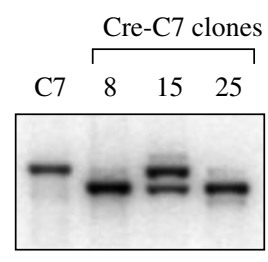

$\mathrm{C}$

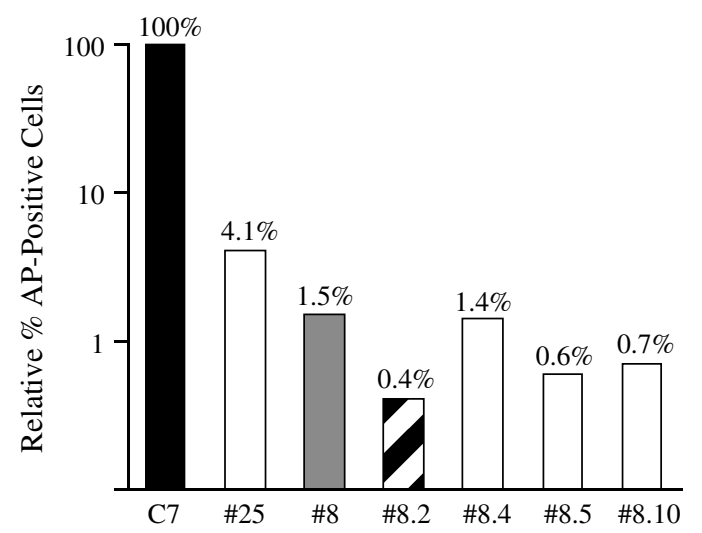

B

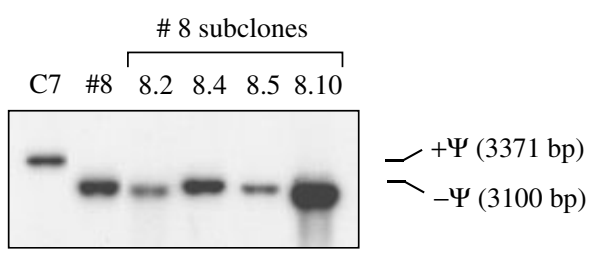

$\mathrm{D}$

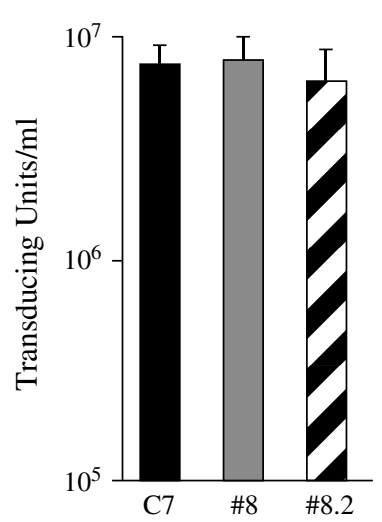

Figure 3. C7-cre cells efficiently excise the loxP-flanked packaging signal and reduce production of helper viruses. C7 and C7-cre cell lines were inoculated with equal amounts of cell lysates (A) or purified virus (B) containing $(+)$ loxP virus $[\mathrm{Adlox} P(+) \mathrm{PolAP}]$ and analyzed $48 \mathrm{~h}$ later. (A) and (B) Southern analysis of viral DNA digested with HindIII and probed with the Ad5 left-end fragment (0-0.5 mu). Samples from C7 cells, C7-cre clones and subclones are indicated. Predicted fragment sizes are noted to the right of panel B. (C) Viral lysates from the C7 and C7-cre cell lines were also analyzed using a colorimetric assay for alkaline phosphatase to determine the relative amounts of virus produced. Data is presented as the percent alkaline phosphatase positive EcR-293 cells, relative to the number of alkaline positive cells obtained with virus grown on C7 cells (defined as 100\%). (D) Viral titers of (-)loxP virus (AdL60) produced in C7 and C7-cre cell lines \#8 and \#8.2. Error bars = SEM of three experiments. Using Student's t-test, titers between the cre and $\mathrm{C} 7$ cells were not significantly different at a $95 \%$ confidence level

\section{Growth of gutted Ad vectors using C7-cre cells}

The C7-cre cell line \#8 (C7-cre8) was subsequently used to optimize a procedure for amplification of gutted adenoviruses. To simplify titering, we used the gutted adenoviral vector GE $\beta$ Dys, which contains a $\beta$-gal cDNA under the control of the ecdysoneinducible promoter, as well as a full-length dystrophin expression cassette (Figure 1B; G. Salvatori, M. Hauser and J. S. Chamberlain, in preparation). Expansion of this virus can therefore be monitored using the EcR-293 colorimetric assay system similar to that for the alkaline phosphatase reporter gene in our helper viruses. Gutted and helper virus production can be assessed in parallel following each round of amplification. Furthermore, $\mathrm{GE} \beta$ Dys contains a loxP site between the L-ITR and the packaging signal. This loxP site ensures that the packaging signal of the helper virus retains two loxP sites in the event that homologous recombination occurs between the sequences of the gutted and helper virus packaging signals [7].

To determine the optimal moi of both helper and gutted viruses that would maximize the yield of gutted virus in the C7-cre cells, we evaluated viral yield after infecting 60-mm dishes with varying amounts of each virus, but always using a helper to gutted transforming unit ratio $(\mathrm{H} / \mathrm{G})$ of either 5 or 1 . The gutted virus GE $\beta$ Dys was derived from a CsCl-purified stock from which the integrity and helper contamination were checked by Southern and quantitative PCR analyses, respectively; the helper virus was added from a CsCl-purified stock to achieve the desired moi. Figure 4 shows the viral yields from these infections and demonstrates that maximum yields of gutted virus were produced when the input helper virus moi was 5 times greater than that of the gutted virus (H5/G1, H25/G5, or H50/G10). An exception was noted when the total input virus moi was 300 . In this case, the yields of helper and gutted viruses were equal suggesting that the cre-recombinase levels were insufficient to process efficiently such large amounts of input virus. It is interesting to note that, although the overall yields were lower with a H/G ratio of 1.0 then with a ratio of 5.0, equal input ratios of the gutted and helper viruses achieved the best relative yield of the two vectors, except at the highest input moi tested (50). These data again suggested that the total viral load had exceeded the ability of the C7-cre cells to excise efficiently the viral packaging signal. Based on these data, we selected an input moi ratio of $5(\mathrm{H} / \mathrm{G})$ for subsequent experiments.

To examine growth of the gutted virus during serial passages, the gutted plasmid pGE $\beta$ Dys was transfected into C7-cre8 cells and subsequently infected with the helper virus $\mathrm{Ad}(+) \operatorname{lox} \mathrm{P}(+)$ PolAP (see 'Materials and 


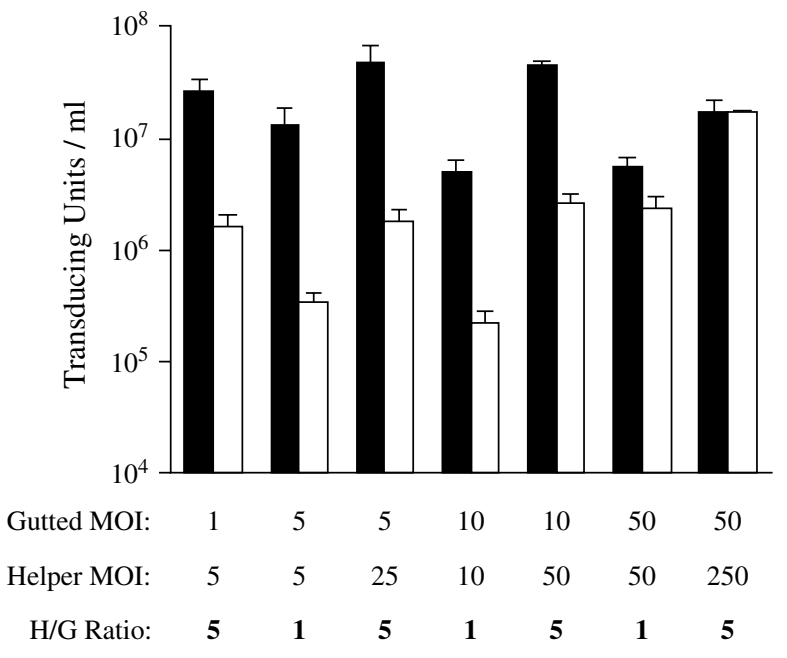

Figure 4. Yield of gutted adenovirus changes with various amounts of input gutted and helper viruses. $60-\mathrm{mm}$ dishes of C7-cre8 cells were infected with moi's as indicated of gutted and helper viruses. $48 \mathrm{~h}$ post-infection, viral yields were determined using the colorimetric assay by counting alkaline phosphatase and $\beta$-gal positive EcR-293 cells $(\mathrm{tu} / \mathrm{ml})$. Shaded bars represent yield of gutted virus; unshaded bars represent yield of helper virus. Error bars $=$ SEM of three experiments

methods'). The gutted virus GE $\beta$ Dys was recovered, or rescued, from this plasmid transfection (termed passage zero or P0) as a cell lysate and subsequently expanded to $100 \times 150 \mathrm{~mm}$ tissue culture dishes during three passages (Figure 5A). The amount of gutted virus increased with each of the first two passages (P1 and P2), then reached a plateau between $10^{7}$ and $10^{8} \mathrm{tu} / \mathrm{ml}$, while the amount of helper virus rescued after each passage remained between $10^{6}$ and $10^{7} \mathrm{tu} / \mathrm{ml}$. Following P3, the $(+)$ loxP helper virus titers were $3-4 \%$ that of the gutted virus. Purification of the gutted virus through two continuous $\mathrm{CsCl}$ gradients reduced the helper contamination to less than $1 \%$ (the lowest we have achieved is $0.2 \%$ as determined by realtime PCR). It is possible that the total amount of gutted virus could be increased even beyond this yield since a lower input moi of gutted virus (moi 1 vs. 5) results in similar yields in the lysate, thus allowing one to infect more dishes starting with a given stock of virus or lysate (Figure 4). Similar results were observed for the C7-cre8.2 subline (data not shown).

Figure 5B shows the theoretical yield if all of the infected lysate generated in the first two passages were used at the maximum dilution to infect additional plates at an optimal moi. The lysate produced in P2 typically yields enough gutted virus to inoculate $200 \times 150 \mathrm{~mm}$ dishes of C7-cre cells (at a gutted moi of 2 ) with a potential yield of $\sim 2 \times 10^{11} \beta$-gal transforming units (tu). In summary, the use of the C7-cre cell lines with a helper virus carrying loxP sites on each side of its packaging signal not only reduces the helper virus contamination obtained with C7 cells, but also reduces to only 3-4 the number of passages necessary to reach the virus production plateau. This limit has been reported previously to be approximately $10^{7} \mathrm{tu} / \mathrm{ml}$ of gutted virus with Cre-293 cells [6], whereas
A

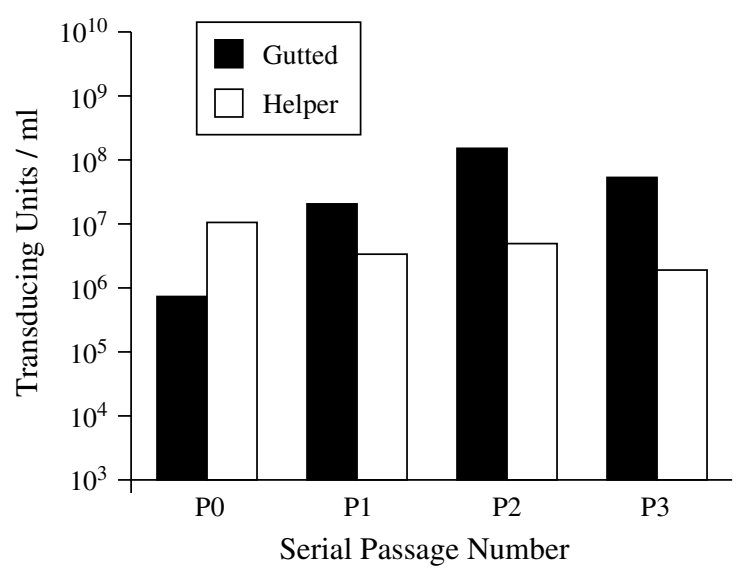

B

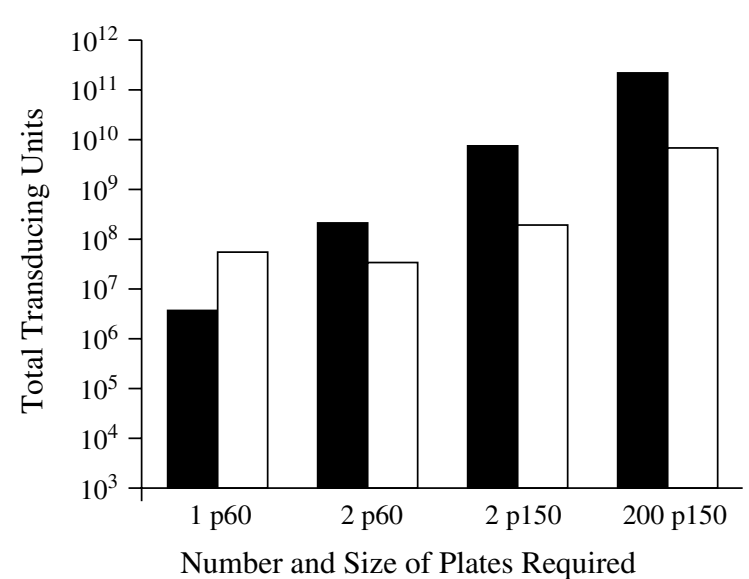

Figure 5. C7-cre cells support amplification of gutted virus while efficiently preventing growth of the $(+)$ loxp helper virus. (A) For rescue of gutted virus, C7-cre cells (clone $\# 8$ ) in a $60-\mathrm{mm}$ dish were transfected with $10 \mu \mathrm{g}$ of pGE $\beta$ Dys followed by infection with $(+)$ loxP helper virus [Ad(+)loxP(+)PolAP]. Subsequent passages (P1, P2, P3) were inoculated with GE $\beta$ Dys at an moi of 1-2 tu/cell and (+)loxP helper virus at a moi of 5, except for P3, which used a helper moi of 3 . P1 $=100-\mathrm{mm}$ dish, P2 $=150-\mathrm{mm}$ dish and P3, the final passage, was in $100 \times 150 \mathrm{~mm}$ dishes. (B) Maximal theoretical yield after three passages of GE $\beta$ Dys gutted virus using $(+)$ lox $\mathrm{P}$ helper virus

the C7-cre8 lines routinely support plateau growth at $>10^{8} \mathrm{tu} / \mathrm{ml}$ (Figures $5 \mathrm{~A}$ and $6 \mathrm{~A}$ ).

\section{Growth of gutted Ad vectors with an E1-, E2b- and E3-deleted helper virus}

The gutted Ad system described above allows vectors to be produced with helper virus contamination levels of $<1 \%$, as assayed by quantitative, real-time PCR. Nonetheless, if very high doses of gutted Ad vectors were to be delivered in vivo, the residual first-generation Ad helper could lead to undesired immunological consequences. We have shown previously that the C7 cell line is able to support efficient, high-titer growth of replication-defective E2bdeleted Ad vectors that display a profound reduction in 


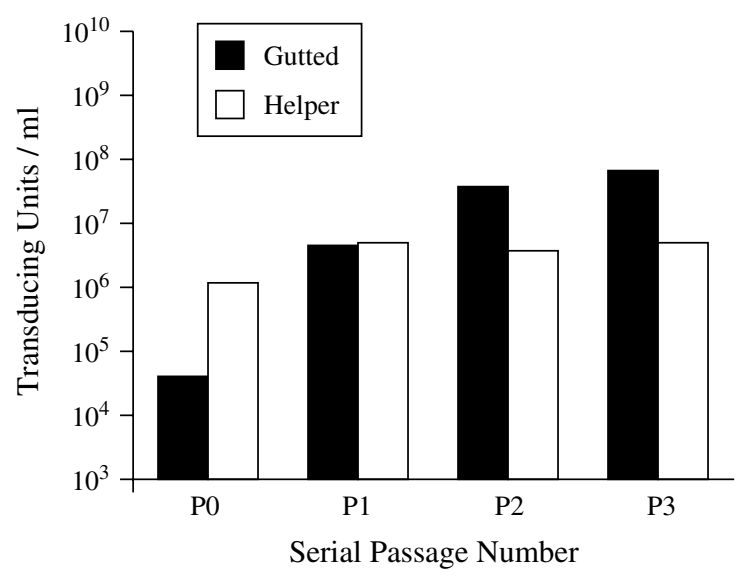

B

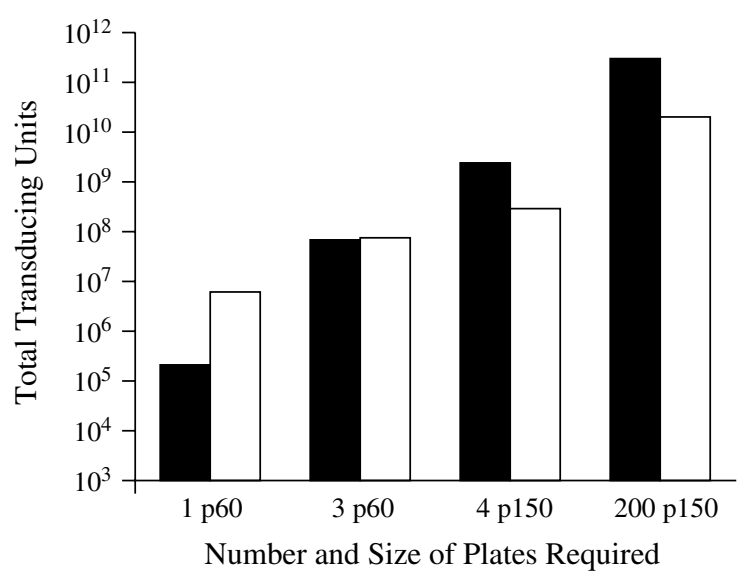

Figure 6. Propagation of GE $\beta$ Dys using the $(+) \operatorname{lox} \mathrm{P} \Delta \mathrm{Pol}$ helper virus in C7-cre cells. (A) GE $\beta$ Dys was rescued in C7-cre cells as described in Figure 5 with the following exceptions: the helper virus $[\mathrm{Ad}(+) \operatorname{lox} \mathrm{P}(+) \mathrm{PolAP}]$ lacked the viral polymerase gene, passage P2 used $2 \times 60 \mathrm{~mm}$ dishes, and P3 used $2 \times 150 \mathrm{~mm}$ dishes. (B) Maximal theoretical yield after three passages of $\mathrm{GE} \beta$ Dys gutted virus using the (+)loxP $\Delta$ Pol helper virus

viral gene expression both in vitro and in vivo [13]. E2bdeleted helper viruses also display increased persistence and reduced cellular toxicity in vivo [17]. We therefore explored whether Ad vectors with deletions of the E2b gene encoding DNA polymerase could support high-titer production of gutted Ad vectors in C7-cre cells. For these studies we constructed a helper virus that contains loxP sites flanking the packaging signal and which lacks the polymerase gene, Ad(+)loxP $\triangle$ PolAP (Figure 1A). This helper virus was used to expand GE $\beta$ Dys in C7-cre cells. Using a helper virus moi of 5 , the amount of gutted virus rescued from the transfection/infection with this $\Delta$ Pol helper virus was $\sim 5 \times 10^{4} \mathrm{tu} / \mathrm{ml}$ (Figure 6A). This yield was 10 -fold lower than the titer of gutted virus produced with the helper virus that contained the Pol gene, suggesting that the $\Delta$ Pol helper was less able to support gutted Ad growth, possibly because it displayed less robust replication. Indeed, these results are consistent with burst assay experiments that showed a $55 \%$ reduction in titer of $\Delta \mathrm{Pol}$ helper virus compared with the (+)Pol helper virus when grown in C7 cells (data not shown).

Despite the slight growth disadvantage of the $\Delta \mathrm{Pol}$ helper virus, titers of nearly $10^{8} \mathrm{tu} / \mathrm{ml}$ of gutted virus were achieved after three serial passages, similar to yields obtained with the (+)Pol helper (Figure 6A). In addition, the theoretical yield following expansion of the entire lysate would be the same, $>10^{11}$ tu (Figure 6B).

\section{Discussion}

Gutted adenoviral vector technology is a promising approach to reducing the immunological barriers that have limited the use of conventional Ad vectors for in vivo gene transfer. The lack of viral genes combined with a large cloning capacity enables gutted vectors to deliver transgene products in tightly regulated or tissue-specific patterns, reducing the propensity to activate immune effector cells [5,23-29]. Nonetheless, the need to use a helper virus to grow a gutted vector complicates the use of this system by introducing low levels of viral gene contamination. In addition, the requirement for serial passaging to obtain high-titer stocks of vector raises the risk of vector rearrangement during growth. The modified helper viruses and packaging cell lines we describe here provide several important improvements to gutted vector technology that should help reduce these concerns by enabling robust growth of vectors in C7 cells using E2bdeleted helper viruses whose growth can be controlled by cre recombinase.

Since C7 cells stably express the E2b genes Pol and pTP, they should allow the use of a helper virus deleted for either, or both, genes. We have shown that a helper virus deleted for the Ad DNA polymerase gene can support high-titer gutted Ad vector growth (Figure 6). Although slightly less efficient than a polymerase-positive helper virus, the $\Delta$ Pol helper virus was still able to generate at least $10^{11}$ tu $\left(\sim 10^{13}\right.$ particles $)$ of gutted virus in only three serial passages. Titers of $10^{7}$ tu of gutted vector/ml in crude cellular lysates were generated in only two passages, in contrast to the previous need for six to seven passages reported by us in 293 cells, and by Parks et al. using Cre-293 cells and a polymerase positive helper virus $[3,6]$. We feel the use of a $\Delta$ Pol helper can offer several significant advantages. Polymerase-deleted Ad vectors have been shown to be replication-defective in the absence of a trans-complementing cell line [13]. This replication defect results in a $4-5 \log$ reduction in viral late gene expression, and enables a significant prolongation of vector retention in vivo as well as reduced toxicity when delivered to the liver $[13,17]$. In addition, multiply deleted Ad vectors have been shown to display a significantly lowered propensity to recombine with viral genes in packaging cell lines to generate replicationcompetent genomes [30-33]. This latter risk can be further reduced by inserting sequences into the $\mathrm{E} 3$ region 
to make the helper genome too large to be packaged if it acquires E1 sequences from the packaging cells [6]. The $\Delta$ Pol helper we describe in Figure 1 also incorporated this feature.

The levels of Pol and pTP expressed by C7-cre cells also support efficient conversion of plasmid substrates into replicating Ad genomes [15], and high-titer viral yields can be obtained starting with both linear and plasmid-embedded versions of the helper vector [5]. The resulting improvements in gutted virus titer following initial transfection/infections (P0) enables infection of packaging cells with the optimal input moi of gutted vector (between 1 and 5) as early as P1 or P2. Since cre-recombinase expression prevents helper virus propagation, an input moi of $1-5$ for the gutted vector is critical for maximal yields as it ensures that most of the cells infected with helper virus are co-infected with gutted vector. The ability to infect cells with optimal titers early in the preparations results in high-titer gutted vector yields with fewer serial passages, not only allowing faster production of gutted virus, but also reducing the number of rounds of vector growth, thereby reducing the opportunities for a vector to rearrange or recombine. In theory, it may be possible to reduce the number of passages required for gutted virus growth even further. We have recently shown that conversion of gutted vector DNA into replicating viral DNA can be enhanced by performing the transfection using gutted DNA that has been covalently modified to contain terminal protein at the ends of the genome [16]. This form of the substrate is in a conformation that more closely models the optimal template necessary for initiation of viral DNA replication.

Regardless of whether the helper contains or lacks the polymerase gene, it appears difficult to achieve gutted virus titers higher than $10^{8} \mathrm{tu} / \mathrm{ml}$ of lysate. In our experience, the titer of the gutted virus increases only slightly as the input gutted moi is increased from 1 to 5 . The use of an input gutted moi higher than 5 does not increase the gutted titer and can lead to reduced yields, even with the use of a higher helper moi. In contrast to the growth of a first- or second-generation adenovirus, gutted virus amplification requires a fine balance between the gutted and the helper ratios. We suspect that too much gutted virus inhibits replication of the helper virus by competing with cellular factors necessary for viral DNA replication. Increasing the amount of helper virus in parallel with the input gutted moi also does not lead to increased yields since high helper loads lead to a cellular cytopathic effect before efficient replication of the gutted vector can occur.

Using the cre-loxp system, the main concern with gutted vector growth is the generation of a packagable helper virus that would contaminate the final gutted virus preparation. One event that can give rise to packagable helper virus is a recombination that leads to replacement of the packaging signal of the helper by the packaging signal of a gutted vector that has no loxp sites. This possibility has been shown to be reduced considerably by either placing a loxP site between the left ITR and the packaging signal of the gutted virus [7] or by limiting contiguous homology between the two packaging signals by replacing the eight ambiguous nucleotides within each A-element with sequences taken from a different A-element [8]. In the gutted virus shown in Figure 1, we added a single $\operatorname{lox} \mathrm{P}$ site, although there is no reason that the packaging signal of our $\Delta \mathrm{Pol}$ helper viruses could not also be modified to reduce potential recombination.

The system we have described here thus incorporates novel and established technologies into reagents for gutted virus production that should allow transfer of a variety of genes and regulatory elements into cells in vivo. We have used this system to produce gutted adenovirus vectors able to transduce skeletal muscle of dystrophindeficient $m d x$ mice with full-length (426 kDa) dystrophin resulting in physiological improvement (C. DelloRusso, C. Barjot, J. M. Scott, J. S. Chamberlain et al., submitted). The large cloning capacity of these vectors, combined with the additional safety and utility afforded by the C7cre system and E2b-deleted helper viruses, may further increase the potential for gene therapy of a variety of diseases.

\section{Acknowledgements}

We thank Nuria Morral, Christiana DelloRusso, Robert Crawford, and Michael Blankenship for helpful discussions. Supported by grants from the National Institutes of Health (AG15434) and the Muscular Dystrophy Association (USA) (to JSC). CB was supported in part by a post-doctoral fellowship from the Association Français Contre les Myopathies.

\section{References}

1. Hitt MM, Graham FL. Adenovirus vectors for human gene therapy. Adv Virus Res 2000; 55: 479-505.

2. Mitani K, Graham FL, Caskey CT, et al. Rescue, propagation, and partial purification of a helper virus-dependent adenovirus vector. Proc Natl Acad Sci U S A 1995; 92: 3854-3858.

3. Kumar-Singh R, Chamberlain JS. Encapsidated adenovirus minichromosomes allow delivery and expression of a $14 \mathrm{~kb}$ dystrophin cDNA to muscle cells. Hum Mol Genet 1996; 5: 913-921.

4. Fisher KJ, Choi H, Burda J, et al. Recombinant adenovirus deleted of all viral genes for gene therapy of cystic fibrosis. Virology 1996; 217: 11-22.

5. Hartigan-O'Connor D, Barjot C, Salvatori G, et al. Growth and purification of gutted adenoviral vectors. Methods Enzymol 2002; in press.

6. Parks RJ, Chen L, Anton M, et al. A helper-dependent adenovirus vector system: removal of helper virus by cremediated excision of the viral packaging signal. Proc Natl Acad Sci U S A 1996; 93: 13 565-13570.

7. Hardy S, Kitamura M, Harris-Stansil T, et al. Construction of adenovirus vectors through Cre-lox recombination. J Virol 1997; 71: $1842-1849$

8. Sandig V, Youil R, Bett AJ, et al. Optimization of the helperdependent adenovirus system for production and potency in vivo. Proc Natl Acad Sci U S A 2000; 97: 1002-1007.

9. Zou L, Zhou H, Pastore L, et al. Prolonged transgene expression mediated by a helper-dependent adenoviral vector (hdAd) in the central nervous system. Mol Ther 2000; 2: 105-113. 
10. Bristol JA, Shirley P, Idamakanti N, et al. In vivo dose threshold effect of adenovirus-mediated factor VIII gene therapy in hemophiliac mice. Mol Ther 2000; 2: 223-232.

11. Tao N, Gao GP, Parr M, et al. Sequestration of adenoviral vector by Kupffer cells leads to a nonlinear dose response of transduction in liver. Mol Ther 2001; 3: 28-35.

12. Schnell MA, Zhang Y, Tazelaar J, et al. Activation of innate immunity in nonhuman primates following intraportal administration of adenoviral vectors. Mol Ther 2001; 3: $708-722$.

13. Amalfitano A, Hauser $\mathrm{MA}, \mathrm{HuH}$, et al. Production and characterization of improved adenovirus vectors with the E1, E2b, and E3 genes deleted. $J$ Virol 1998; 72: 926-933.

14. Amalfitano A, Chamberlain JS. Isolation and characterization of packaging cell lines that co-express the adenovirus E1, DNA polymerase, and preterminal proteins: implications for gene therapy. Gene Ther 1997; 4: 258-263.

15. Hartigan-O'Connor D, Amalfitano A, Chamberlain JS. Improved production of gutted adenovirus in cells expressing adenovirus preterminal protein and DNA polymerase. J Virol 1999; 73: 7835-7841.

16. Hartigan-O'Connor D, Barjot C, Salvatori G, et al. Efficient rescue of gutted adenovirus genomes allows rapid production of concentrated stocks without negative selection. Hum Gene Ther 2002; in press.

17. Hu H, Serra D, Amalfitano A. Persistence of an [E1-, polymerase-] adenovirus vector despite transduction of a neoantigen into immune-competent mice. Hum Gene Ther 1999; 10: $355-364$.

18. Amalfitano A, Begy CR, Chamberlain JS. Improved adenovirus packaging cell lines to support the growth of replicationdefective gene-delivery vectors. Proc Natl Acad Sci U S A 1996; 93: 3352-3356.

19. O'Gorman S, Dagenais NA, Qian M, et al. Protamine-Cre recombinase transgenes efficiently recombine target sequences in the male germ line of mice, but not in embryonic stem cells. Proc Natl Acad Sci U S A 1997; 94: 14602-14607.

20. MacGregor GM, Nolan GP, Fiering S, et al. Use of E. coli LacZ ( $\beta$ galactosidase) as a reporter gene. In Methods Molecular Biology, vol 7. Gene Transfer and Expression Protocols, Murray EJ (ed.). Human Press Inc.: Clifton, NJ, 1991; 217-225.

21. Hirt B. Selective extraction of polyoma DNA from infected mouse cell cultures. J Mol Biol 1967; 26: 365-369.
22. Graham FL, Smiley J, Russell WC, et al. Characteristics of a human cell line transformed by DNA from human adenovirus type 5. J Gen Virol 1977; 36: 59-72.

23. Morral N, Parks R, Zhou H, et al. High doses of a helperdependent adenoviral vector yield supraphysiological levels $\alpha 1$-antitrypsin with negligible toxicity. Hum Gene Ther 1998; 9: 2709-2716.

24. Chen HH, Mack LM, Kelly R, et al. Persistence in muscle of an adenoviral vector that lacks all viral genes. Proc Natl Acad Sci U S A 1997; 94: 1645-1650.

25. Morsy MA, Gu M, Motzel S, et al. An adenoviral vector deleted for all viral coding sequences results in enhanced safety and extended expression of a leptin transgene. Proc Natl Acad Sci U S A 1998; 95: 7866-7871.

26. Schiedner G, Morral N, Parks RJ, et al. Genomic DNA transfer with a high-capacity adenovirus vector results in improved in vivo gene expression and decreased toxicity. Nat Genet 1998; 18: $180-183$.

27. Pastore L, Morral N, Zhou HS, et al. Use of a liver-specific promoter reduces immune response to the transgene in adenoviral vectors. Hum Gene Ther 1999; 10: 1773-1781.

28. O'Neal WK, Zhou H, Morral N, et al. Toxicity associated with repeated administration of first-generation adenovirus vectors does not occur with a helper-dependent vector. Mol Med 2000; 6: 179-195.

29. Hartigan-O'Connor D, Kirk CJ, Crawford R, et al. Immune evasion by muscle specific gene expression in dystrophic muscle. Mol Ther 2001; 4: 525-533.

30. Yeh P, Dedieu JF, Orsini C, et al. Efficient dual transcomplementation of adenovirus E1 and E4 regions from a 293-derived cell line expressing a minimal E4 functional unit. $J$ Virol 1996; 70: 559-565.

31. Fallaux FJ, Bout A, van der Velde I, et al. New helper cells and matched early region 1-deleted adenovirus vectors prevent generation of replication-competent adenoviruses. Hum Gene Ther 1998; 9: 1909-1917.

32. Lusky M, Christ M, Rittner K, et al. In vitro and in vivo biology of recombinant adenovirus vectors with E1, E1/E2A, or E1/E4 deleted. $J$ Virol 1998; 72: 2022-2032.

33. Gorziglia MI, Lapcevich C, Roy S, et al. Generation of an adenovirus vector lacking E1, E2a, E3, and all of E4 except open reading frame 3. $J$ Virol 1999; 73: 6048-6055. 